\title{
Surface energy analysis (SEA) study of hyaluronan powders
}

\author{
Lubomír Lapčík $^{1,2,3 *}$, Eva Otyepková ${ }^{1}$, Barbora Lapčíkováa $^{2,3}$, Michal Otyepka $^{1}$
}

${ }^{1}$ Regional Centre of Advanced Technologies and Materials, Department of Physical Chemistry, Faculty of Science, Palacky University, 17. listopadu 12, 77146 Olomouc,

Czech Republic

${ }^{2}$ Tomas Bata University in Zlin, Faculty of Technology, Institute of Foodstuff Technology, nám. T.G. Masaryka 5555, 76005 Zlín, Czech Republic

${ }^{3}$ Center of Polymer Systems, Tomas Bata University in Zlin, Faculty of Technology,

Nam. T.G. Masaryka 5555, Zlin, Czech Republic

${ }^{*}$ Corresponding author.

E-mail address: lapcikl@seznam.cz

Key words: hyaluronan, powder, wetting, surface energy, distribution, surface energy analysis

\begin{abstract}
Results of inverse gas chromatography adsorption/desorption experiments of selected probes on sodium hyaluronate powder material are presented. It was found that a dominating was a dispersive surface energy part thus indicating low polarity character of the studied HA powder. For $0 \%$ coverage $30 \mathrm{~mJ} / \mathrm{m}^{2}$ total surface energy was found. There was found a relatively high inhomogeneity of the surface structure of the studied polymer powder. A total surface energy distribution was ranging from 10 to $34 \mathrm{~mJ} / \mathrm{m}^{2}$ with maximum at $18.5 \mathrm{~mJ} / \mathrm{m}^{2}$. It was similarly as in the previous case of surface energy profile controlled by dispersive part. By measuring free energy profiles dependencies for selected probe molecules of different polarity there was found approximately seven-fold higher energy content $(15 \mathrm{~kJ} / \mathrm{mol})$ in comparison to dichloromethane $(2 \mathrm{~kJ} / \mathrm{mol})$. There were determined work of cohesion and work of adhesion (water) on HA surface.
\end{abstract}

\section{Introduction}

The properties of surfaces and interfaces characterized by surface or interfacial tension and surface energy are of a growing importance in recent years. These properties are joined with many phenomena concerning adhesion, wetting, spreading and wicking which express themselves in everyone's daily life, natural processes as well as in huge amount of industrial applications such as coating, printing, lubrication, composite or mineral processing, textile and wood finishing, oil recovery, painting, highly absorbent materials and adhesives to name a few. These processes involve various materials for instance biopolymers [1-4], synthetic polymers, wood, paper, stone, soils, cereals and textile which could cover all possible types of surfaces: polar, non-polar, much more often rough than smooth or even porous and this may bring many obstructions to their surface characterization. 
There are several well-known techniques of contact angle measurement on flat and smooth surfaces e.g. sessile drop or adhering gas bubble method and Wilhelmy method [5]. Nevertheless, most of the real surfaces are not ideal but rough and heterogeneous and many materials are available only in the form of powders and fibres. It may be possible to compress these particles to obtain flat surface but such system could provide different contact angle values because the material undergoes structural and possibly also significant chemical changes. Therefore, it would be more suitable to measure contact angles directly on the original surface.

Despite the difficulties, there are several methods which are applicable to powder and fibrous materials [5-10]. The most popular is capillary rise method, thin-layer wicking and the use of Wilhelmy method [5]. Furthermore, the measurements seem to yield also an additional notion of pore size and structure of the material [5], although it does not provide so extensive information such as mercury porosimetry. However, at the present time, a new surface energy analysis technique based on inverse gas chromatography has been found to be very effective in characterization of wetting phenomena on powders and fibres [6-10].

Hyaluronan (HA), a high molecular weight biopolysaccharide, was discovered by Meyer and Palmer in 1934 in the vitreous humour of cattle eyes [1]. HA is a member of a group of similar polysaccharides that have been termed "connective tissue polysaccharides", "mucopolysaccharides", or "glycosaminoglycans". These polysaccharides include chondroitin sulfate, dermatan sulfate, keratan sulfate, heparan sulfate, and heparin. HA is a linear, unbranched polymer. Meyer and co-workers found HA to be composed of a repeating disaccharide that consists of $N$-acetyl-D-glucosamine (GlcNAc) and D-glucuronic acid (GlcA) linked by a $\beta$ 1-4 glycosidic bond [1]. The disaccharides are linked by $\beta 1-3$ bonds to form the HA chain. In addition to its presence in the vitreous body, HA occurs in many living substrata such as the extracellular matrix and synovial fluids [1-4]. In practise, HA is used in wound healing, because it supports tissue reconstruction. During the first few days of tissue repair, endogeneous HA is the predominant glycosaminoglycan present in wounds and forms the template necessary for reconstruction following injury [1]. HA could be used to enhance the localization of a number of possible drugs within the epidermis/dermis. Such an effect is an obvious advantage if the site of action lies within the skin layers (e.g., antifungal and antibacterial agents) [1]. A detailed knowledge of surface properties of HA is important for practical pharmaceutical application e.g. in drug formulation, construction of wound healing dressings, tablets, capsules, dry powder inhalation formulations etc.

That is why in this study we analyze surface properties of HA using inverse gas chromatography, which is an excellent tool for characterization of surface properties of powder materials.

\section{Methods}

\subsection{Theoretical background}

Surface free energy of a solid can be described as the sum of the dispersive and specific contributions. Dispersive (apolar) interactions, also known as Lifshitz-van der Waals interactions, consist of London interactions which originate from electron density changes but may include both Keesom and Debye interactions $[5,6]$. Other forces influencing the magnitude of surface energy are Lewis acid-base interactions which are generated between electron acceptor (acid) and electron donor (base). They appear in the compounds containing hydrogen bonds - strong secondary bonds between atoms of hydrogen and a highly electronegative element such as $\mathrm{F}, \mathrm{O}, \mathrm{N}$ and $\mathrm{Cl}$ or other compounds interacting with Lewis acids and bases. Details of the widely accepted 
theoretical treatment of the estimation of solid surface free energy by selective wetting measurements are described in detail in our review article [5].

The dispersive component of the surface energy $\gamma_{S}^{D}$ can be calculated from the retention time obtained from inverse gas chromatography measurements of a series of n-alkane probes injected at infinite dilution (concentration within the Henry's portion of the adsorption isotherm) [7]. For evaluation of these dependencies there are used two approaches, the first one according to Schultz et al. [8] (1) and the second one according to Dorris and Gray [9] (2):

$$
R T \ln V_{N}=a\left(\gamma_{L}^{D}\right)^{1 / 2} 2 N_{A}\left(\gamma_{S}^{D}\right)^{1 / 2}+C
$$

where $R$ is the universal gas constant, $N_{A}$ is Avogadro's number, $\gamma_{L}^{D}$ is the dispersive component of surface free energy of the liquid probe, $\gamma_{S}^{D}$ is the dispersive component of the surface free energy of the solid, $V_{N}$ is the retention volume and $C$ is a constant, and

$$
\gamma_{S}^{D}=\frac{\left\lfloor R T \ln \left(V_{N\left(C_{n+1} H_{2 n+4}\right)}\right) / V_{N\left(C_{n} H_{2 n+2}\right)}\right\rfloor}{4 N_{A}^{2} a_{C H_{2}}^{2} \gamma_{C H_{2}}}
$$

where $a_{\mathrm{CH}_{2}}$ is the surface area of a $\mathrm{CH}_{2}$ unit $\left(\sim 0.6 \mathrm{~nm}^{2}\right)$ and $\gamma_{\mathrm{CH}_{2}}$ is its free energy (approximately 35.6 $\left.\mathrm{mJ} / \mathrm{m}^{2}\right)$

The Hildebrand solubility parameter $(\delta)$ provides a numerical estimate of the degree of interaction between different materials. It can be a good indication of solubility, particularly for non polar materials such as many polymers [10]. Materials with similar values of $\delta$ are likely to be miscible. It is defined as the square root of the cohesive energy density:

$$
\delta=\sqrt{\frac{\Delta H_{V}-R T}{V_{m}}}
$$

Materials with similar magnitude of the solubility parameters will be capable to interact between each other what will result in their mutual solvation, swelling or miscibility.

\subsection{Experimental}

Inverse gas chromatography was conducted using a Surface Energy Analyser (SEA) (Surface Measurement Systems, UK). Samples were placed in $4 \mathrm{~mm}$ (internal diameter) columns, to give a total surface 
area of approximately $0.5 \mathrm{~m}^{2}$. The following eluent vapours were passed through the column: Nonane, Octane, Hexane, Heptane. All reagents were obtained from Sigma Aldrich (USA), and were of analytical grade. The injection of vapours was controlled to pass a set volume of eluent through the column to give pre-determined fractional coverage of the sample in the column. The retention time of the vapours by the particles gives an indication of the surface properties of the material, including the surface energy. By gradually increasing the amount of vapour injected, it is possible to build up a surface heterogeneity plot.

Specific surface area measurements were made using a Micromeritics TriStar 3000 surface area and porosity analyser (USA), using the nitrogen BET technique.

Thermo-gravimetry (TG) and differential thermal analysis (DTA) experiments were performed on simultaneous DTA-TG apparatus (Shimadzu DTG 60, Japan). Throughout the experiment, the sample temperature and weight-heat flow changes were continuously monitored. Conditions of measurement: Heat flow $10{ }^{\circ} \mathrm{C} / \mathrm{min}$ and dynamic atmosphere of nitrogen $\left(\mathrm{N}_{2}-50 \mathrm{ml} / \mathrm{min}\right)$, range of temperature measurement was from $40{ }^{\circ} \mathrm{C}$ to $500{ }^{\circ} \mathrm{C}$.

Scanning electron microscopy (SEM) images were captured on a Hitachi 6600 FEG microscope operating in the secondary electron mode and using an accelerating voltage of $1 \mathrm{kV}$.

Hyaluronate (sodium salt, microbial production) in the form of a white powder was of 0.7 to $0.9 \mathrm{MDa}$ molecular weight (CPN, Ltd., Czech Republic). Sample was kept in dry conditions in desiccator (at the ambient temperature of $22^{\circ} \mathrm{C}$ ) for 4 weeks prior to the SEA experiments.

\section{Results and discussion}

Surface properties of HA powders were analysed by several experimental techniques. Specific surface area of HA powders was found to be of $15 \mathrm{~m}^{2} / \mathrm{g}$ as observed by BET technique, determined sample density was of $1.00 \mathrm{~g} / \mathrm{cm}^{3}$. To characterize exact moisture content in the studied samples DTA-TG measurements were performed. These showed gradual sample mass loss with increasing temperature, having three distinct degradation regions (Fig. 1). The first one, characteristic for water loss of $13.72 \mathrm{w} . \%$ in the temperature end of $180{ }^{\circ} \mathrm{C}$ and the second and third region, characteristic with polysacharide degradation with starting temperature of $180{ }^{\circ} \mathrm{C}$ and $300{ }^{\circ} \mathrm{C}$. Observed weight loss for second region was of $38.55 \mathrm{w} . \%$ and of $12.26 \mathrm{w} . \%$ for the third region. Observed relatively high residual mass percentage at $500{ }^{\circ} \mathrm{C}$ of 35.49 w. $\%$ was ascribed to the presence of sodium ions present in the structure, which can form inorganic sub-products in the degradation reaction [11]. A typical shape of the particles of studied HA powder are shown in Fig. 2. These were characteristic with spherical and cylindrical shape of approximately $70 \mu \mathrm{m}$ wide in diameter.

Surface energy profile and its components of studied HA powder based on inverse gas chromatography measurements data are shown in Fig. 3. Total surface energy and dispersive surface energy part coverage dependencies were of a characteristic exponential decrease with increasing coverage from observed $34.0 \mathrm{~mJ} / \mathrm{m}^{2}$ for $0 \%$ coverage for both parameters, to $11.7 \mathrm{~mJ} / \mathrm{m}^{2}$ for total and to $4.9 \mathrm{~mJ} / \mathrm{m}^{2}$ for dispersive surface energy component at $100 \%$ surface coverage as well. The highest energetic sites occupy approximately only $5 \%$ of the HA surface. The significant difference in measured surface energy absolute values at low and high coverage indicates high degree of inhomogeneity between highest surface energy sites having approximately three fold higher absolute value of the surface energy than the lowest energetic sites. The latter SEA based absolute value 
of dispersive surface energy calculated by Schultz method [8], very well correlate with the published data obtained from contact angle measurements of apolar dispersive component of the surface tension which was found to be $39.8 \mathrm{~mJ} / \mathrm{m}^{2}$ [12]. As shown in Fig. 3, polar acid-base part of the surface energy coverage dependency is characteristic with minor linear increase with increasing coverage ranging from $0.7 \mathrm{~mJ} / \mathrm{m}^{2}$ for $0 \%$ coverage up to $6.8 \mathrm{~mJ} / \mathrm{m}^{2}$ for $100 \%$ coverage. Obtained results indicate dominance of a dispersive part of the surface energy, thus suggesting low polarity character of the studied sample. The surface energy distribution is characteristic with narrow distribution of the total surface energy ranging from 10 to $34 \mathrm{~mJ} / \mathrm{m}^{2}$ with the maximum at $18.5 \mathrm{~mJ} / \mathrm{m}^{2}$ (Fig. 4). The total surface energy distribution is controlled by distribution of the dispersive part which is ranging from $3.3 \mathrm{~mJ} / \mathrm{m}^{2}$ to $34.1 \mathrm{~mJ} / \mathrm{m}^{2}$. It is evident, that the polar surface active sites are of relatively low energy, again documenting the low polarity of sample surface. On the other hand, the dispersive part surface energy distribution is of more wide character, reflecting higher number of structural elements being responsible for this behaviour.

In most material characteristics of formulation components it is usually assumed that the surfaces of materials reflect the inherent nature of the studied substance. However, the surface characteristics of a material can be altered by exposing different crystal planes or by contamination of surfaces with foreign materials (e.g. from the ambient atmosphere) [13]. To verify degree of surface contamination effects on surface energy analysis data, e.g., by adsorbed water molecules and gaseous molecules from the ambient air atmosphere, there was, prior to the next experiment, the studied HA sample dried under the vacuum $\left(50 \mathrm{mbar}, 25^{\circ} \mathrm{C}\right)$ to detach latter contaminants from the sample surface. Surface energy distributions (after vacuum drying) indicate dominance of a dispersive part contribution to the total surface energy ranging from $33 \mathrm{~mJ} / \mathrm{m}^{2}$ to $49 \mathrm{~mJ} / \mathrm{m}^{2}$ with total surface energy distribution reaching its maximum at $52 \mathrm{~mJ} / \mathrm{m}^{2}$ (Fig. 5). When compared with results shown in Fig. 4 the latter contaminant free data were of approximately twice energetic. However their relative occupancy remains at the same area increment level of $0.4 \%$. Distribution of acid-base part of surface energy of surface "decontaminated" sample was ranging from 1 to $8 \mathrm{~mJ} / \mathrm{m}^{2}$, with relatively high area increment occupancy of 2.5 $\%$.

Specific acid-base free energy distributions for applied selected probes reflect the ratio of structural components of the donor-acceptor character present at the HA (s/g) interface (Fig. 6). The broadest distribution was found for acetonitrile ranging from $-11.8 \mathrm{~kJ} / \mathrm{mol}$ up to $15.0 \mathrm{~kJ} / \mathrm{mol}$ specific (acid-base) free energy, the most narrowed one for dichloromethane ranging from -10.8 to $2.3 \mathrm{~kJ} / \mathrm{mol}$. However for all studied probes these were occupying only minor area increments ranging from $0.25 \%$ for dichloromethane, $0.35 \%$ for ethanol, $0.43 \%$ for acetonitrile and $0.53 \%$ for acetone and ethylacetate as well. The majority of the polar character surface constituents are of the energy ranging from -10.6 to $12.8 \mathrm{~kJ} / \mathrm{mol}$.

In Fig. 7 there are shown results of free energy profiles for selected probes with different polarity. It can be seen from the obtained free energy coverage dependencies that the highest reactivity non-polar sites at $0 \%$ coverage are of approximately seven-fold higher energy content $(15 \mathrm{~kJ} / \mathrm{mol})$ in comparison to dichloromethane $(2 \mathrm{~kJ} / \mathrm{mol})$. With increasing surface coverage the linear decrease of the free energy for all probe molecules under study was found reaching a value of $-11 \mathrm{~kJ} / \mathrm{mol}$ for a 0.2 coverage.

Work of HA cohesion and work of adhesion of water on HA sample were calculated from our inverse gas chromatography experiments (Fig. 8). These were characteristic with a linear decrease of all measured quantities (work of cohesion and its components as well as of work of adhesion and its components) with 
increasing coverage. There was a clear confirmation of our previously indicated conclusion of dispersive forces dominance in comparison to the specific interactions. By the application of a spreading coefficient rule the difference of work of adhesion minus work of cohesion if positive, indicates good spreading of water on the studied polymer surface. As shown in Fig. 9 only critical surface coverage regime with limiting spreading of water was found in the range of 7 to $10 \%$ coverage, where a negative value for total work of adhesion difference with total work of cohesion was found. As an addition to the latter results, it can be found from Fig. 9 that the dispersive component difference is negative in the studied coverage regime indicating its negative effect to the interaction with water molecules. However, for a specific part difference there was observed higher absolute value of $14 \mathrm{~mJ} / \mathrm{m}^{2}$ in comparison to the $-10 \mathrm{~mJ} / \mathrm{m}^{2}$ for dispersive part. The latter difference was thus indicating wetting ability of HA powder surface by water molecules to some extent. With respect to the absolute values of free energy extrapolated to the $100 \%$ coverage gives $-80 \mathrm{~kJ} / \mathrm{mol}$ for ethanol and acetone. Observed extrapolated value of $-80 \mathrm{~kJ} / \mathrm{mol}$ indicates dominance of the hydrophobic attraction between individual HA molecules rather than hydrophilic repulsion leading to dispersion or solubilisation in water.

In Fig. 10 there are shown results of the surface coverage dependence of the Hildebrand's solubility parameter $\delta$ for studied HA powder. Its magnitude ranges from $18 \mathrm{MPa}^{0.5}$ for $0 \%$ coverage to $15 \mathrm{MPa}^{0.5}$ for 20 $\%$ coverage. Obtained values of solubility parameter might be one of the parameters allowing us to compare a mutual miscibility of HA with other materials e.g. in aerosol inhalation or wound healing applications.

\section{Conclusions}

It was found in this study that for studied sodium hyaluronate powder with respect to the surface coverage dependence of surface energy a dominating was found a dispersive surface energy part thus indicating low polarity character of the studied HA powder. For $0 \%$ coverage $30 \mathrm{~mJ} / \mathrm{m}^{2}$ total surface energy was found. There was found relatively high inhomogeneity of the surface structure of the studied polymer powder as reflected in the three-fold higher energy content for highest energy sites (at $0 \%$ surface coverage) in comparison to the lowest energy sites as observed for $100 \%$ coverage $\left(11.7 \mathrm{~mJ} / \mathrm{m}^{2}\right)$. A total surface energy distribution was ranging from 10 to $34 \mathrm{~mJ} / \mathrm{m}^{2}$ with maximum at $18.5 \mathrm{~mJ} / \mathrm{m}^{2}$. It was similarly as in the previous case of surface energy profile controlled by dispersive part. There was demonstrated an effect of the surface contamination of studied sample by surrounding atmosphere adhered molecules, thus affecting an absolute values of the determined surface energies distributions. Here after removal of the adsorbed gaseous molecules and moisture acquired from the ambient atmosphere was giving higher absolute values of maximum surface energy by factor of 1.7. However, the overall character and mutual ratio between total, dispersive and acid-base parts was remained constant. By measuring the free energy profiles dependencies for selected probe molecules of different polarity there was found approximately seven-fold higher energy content $(15 \mathrm{~kJ} / \mathrm{mol})$ in comparison to dichloromethane $(2 \mathrm{~kJ} / \mathrm{mol})$. There were determined work of cohesion and work of adhesion (water) on HA surface. It was found that only critical surface coverage regime with limiting spreading of water was found in the range of 7 to $10 \%$ coverage, where a negative value for work of adhesion difference with work of cohesion was found. Finally, the Hildebrand's solubility parameter was found to be ranging from 18 to $15 \mathrm{MPa}^{0.5}$ for $0 \%$ to 20 $\%$ surface coverage regimes. 


\section{Acknowledgements}

Financial support from the Operational Program Research and Development for Innovations - European Regional Development Fund (grants CZ.1.05/2.1.00/03.0058 and CZ.1.05/2.1.00/03.0111) is gratefully acknowledged. Authors would like to express their gratitude for SEM measurements to Dr. K. Šafárová (Palacky University in Olomouc).

\section{References}

[1] Lapčík, Jr. L, Lapčík L, De Smedt S, Demeester J, Chabreček P. Hyaluronan: Preparation, Structure, Properties, and Applications. Chem. Rev. 98 (1998) 2663-2684.

[2] Lapčík L, Kuběna K, Galatík A, Kellö V. Photodegradation of hyaluronic acid and of the vitreous body. Gen. Physiol. Biophys. 9 (1990) 419-429.

[3] Lapčík L, Schurz J. Photochemical degradation of hyaluronic acid by singlet oxygen. Coll. Polym. Sci. 269 (1991) 633-635.

[4] Lapčík, Jr. L, Benešová K, Lapčík L, De Smedt S, Lapčíková B. Chemical modification of hyaluronic acid: alkylation. Int. J. Polym. Anal. Charact. 15 (2010) 486-496.

[5] Gajdošíková R, Lapčíková B, Lapčík L. Surface phenomena and wetting of porous solids. Phys Chem: An Indian J. 6 (2011) 146-162.

[6] Gamble JF, Leane M, Olusanmi D, Tobyn M, Šupuk E, Khoo J, Naderi M. Surface energy analysis as a tool to probe the surface energy characteristics of micronized materials - A comparison with inverse gas chromatography. Int. J. Pharmaceutics 422 (2012) 238-244.

[7] Belgacem MN, Gandini A, Pefferkorn E. (Ed.) Interfacial phenomena in chromatography; Marcel Dekker: New York, p. 145, 1999.

[8] Schultz L, Lavielle C, Martin J. The role of interface in carbon fibre-epoxy composites. J Adhes. 23 (1987) 45-60.

[9] Dorris GM, Gray DG. Adsorption of normal-alkanes at zero surface coverage on cellulose paper and wood fibers. J. Coll. Interf. Sci. 77 (1980) 353-362.

[10] Kwok AY, Qiao GG, Solomon DH. Interpenetrating amphiphilic polymer networks of poly(2-hydroxyethyl methacrylate) and poly(ethylene oxide). Chem. Mater. 16 (2004) 5650-5658.

[11] Villetti MA, Crespo JS, Soldi MS, Pires ATN, Borsali R., Soldi V. Thermal degradation of natural polymers. J. Thermal Anal. Calorimetry 67 (2002) 295-303.

[12] Kaufmann J, Wiegel D, Arnold K. Polar interactions of hyaluronic acid Experiments and Molecular Dynamics Simulation. J. Dispersion Sci. Technol. 19 (1998) 979-1001.

[13] Etzler FM, Uddin MN. Powder technology and pharmaceutical development: $\quad$ particle size and particle adhesion. KONA Powder Particle J. 125 (2013) 125-143. 


\section{Figures and tables charter}

Fig. 1. TG DTA pattern of HA powder under study.

Fig. 2. SEM image of studied HA powder.

Fig. 3. Total surface energy and its components profile plot of HA powder under study: empty circle - total surface energy, empty triangle down - dispersive part of surface energy, empty square - acid - basic part of surface energy.

Fig. 4. Total surface energy and its components distribution plot of HA powder under study. Circle - dispersive part of the surface energy, triangle down - acido-basic part of surface energy, square - total surface energy.

Fig. 5. Total surface energy and its components distribution plot of freeze-dried HA powder. Circle - dispersive part of the surface energy, triangle down - acido-basic part of surface energy, square - total surface energy.

Fig. 6. Specific acid-base free energy distributions of studied HA powder as observed for selected wetting probes: triangle down - ethyl acetate, square - acetone, diamond - acetonitrile, triangle up - ethanol.

Fig. 7. Coverage dependence of free energy profiles of studied HA powder as observed for selected wetting probes: full circle - dichloromethane, empty triangle down - ethyl acetate, full square - acetone, empty diamond - acetonitrile, full triangle up - ethanol.

Fig. 8. Work of cohesion between HA and work of adhesion and its components of water on studied HA powder at $30{ }^{\circ} \mathrm{C}$ for different coverage regimes: empty circle - work of cohesion dispersive part, empty triangle down work of cohesion specific part, empty square - work of cohesion total, full circle - work of adhesion dispersive part, full triangle down - work of cohesion specific, full square - work of adhesion total.

Fig. 9. Difference between work of adhesion of water on HA and work of cohesion of HA and its components as a function of coverage of studied HA powder at $30^{\circ} \mathrm{C}$ : full circle - total, empty triangle down - dispersive, full square - specific.

Fig. 10. Surface coverage dependence of the Hildebrand's solubility parameter $\delta$ of studied HA powder. Full line - linear regression, dashed line $-95 \%$ confidence interval. 


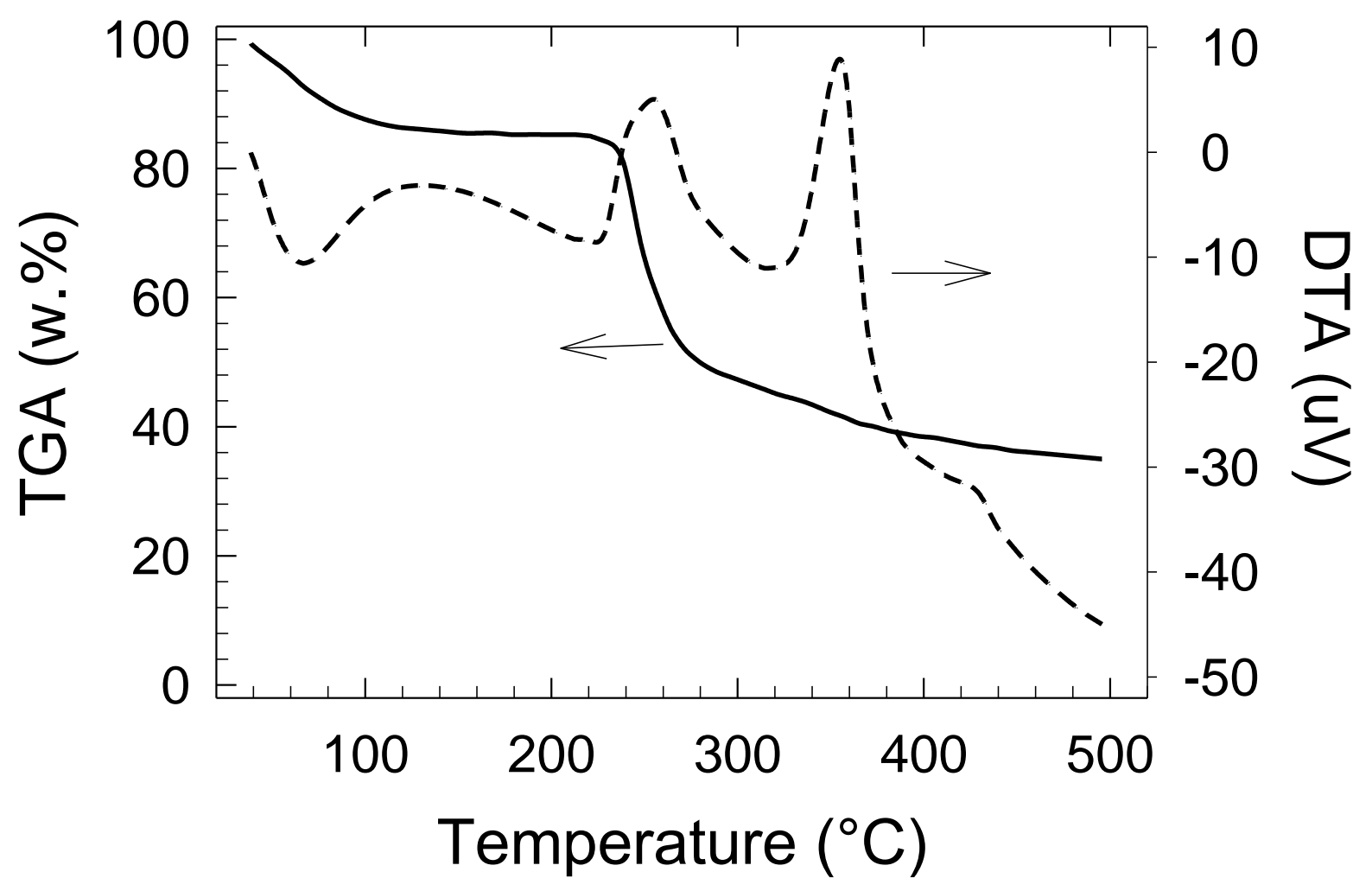

Figure 1. TG DTA pattern of HA powder under study. 




Figure 2. SEM image of studied HA powder. 




Figure 3. Total surface energy and its components profile plot of HA powder under study: empty circle - total surface energy, empty triangle down - dispersive part of surface energy, empty square - acid - base part of surface energy. 


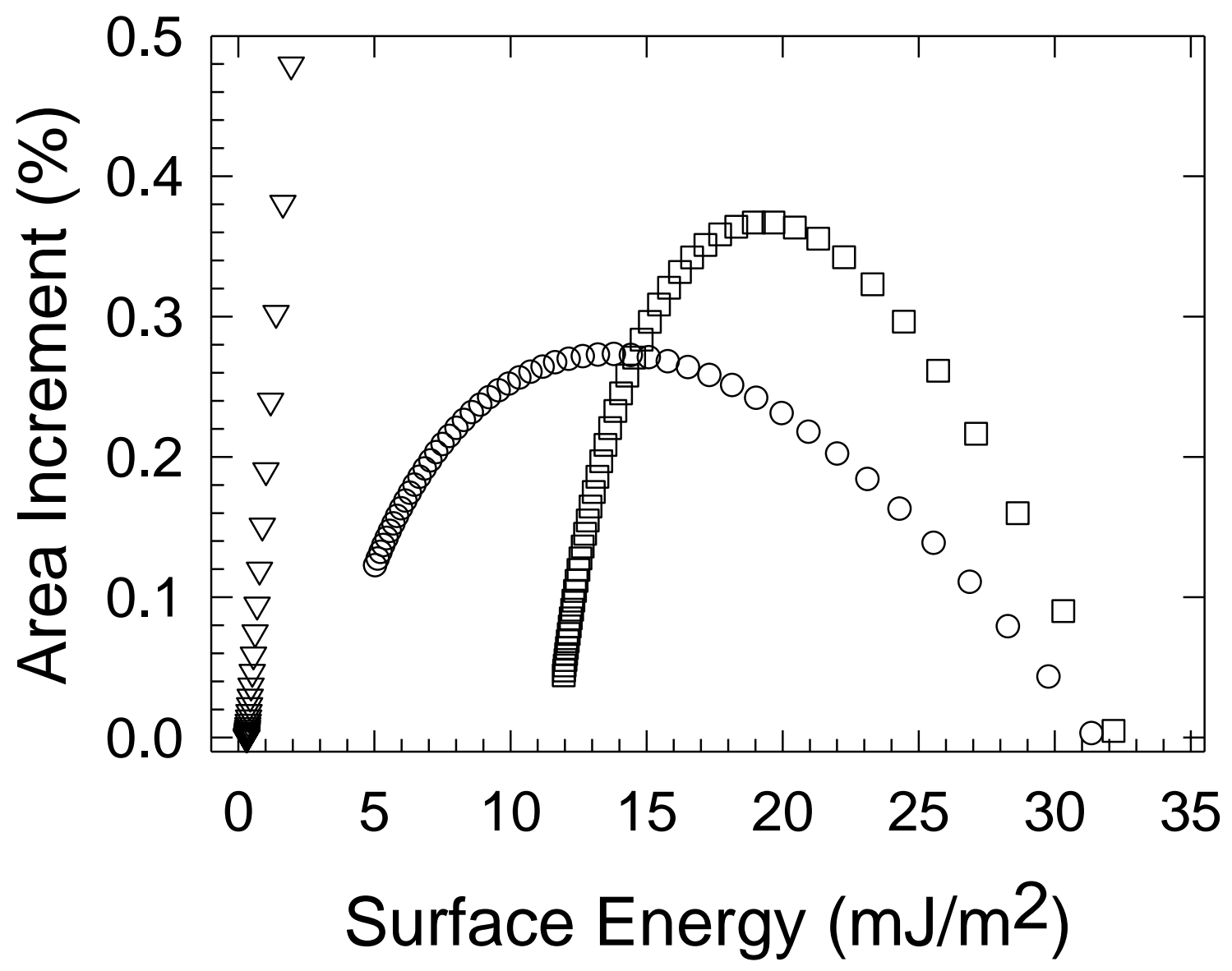

Figure 4. Total surface energy and its components distribution plot of HA powder under study. Circle dispersive part of the surface energy, triangle down - acido-basic part of surface energy, square - total surface energy. 




Figure 5. Total surface energy and its components distribution plot of freeze-dried HA powder. Circle dispersive part of the surface energy, triangle down - acido-basic part of surface energy, square - total surface energy. 


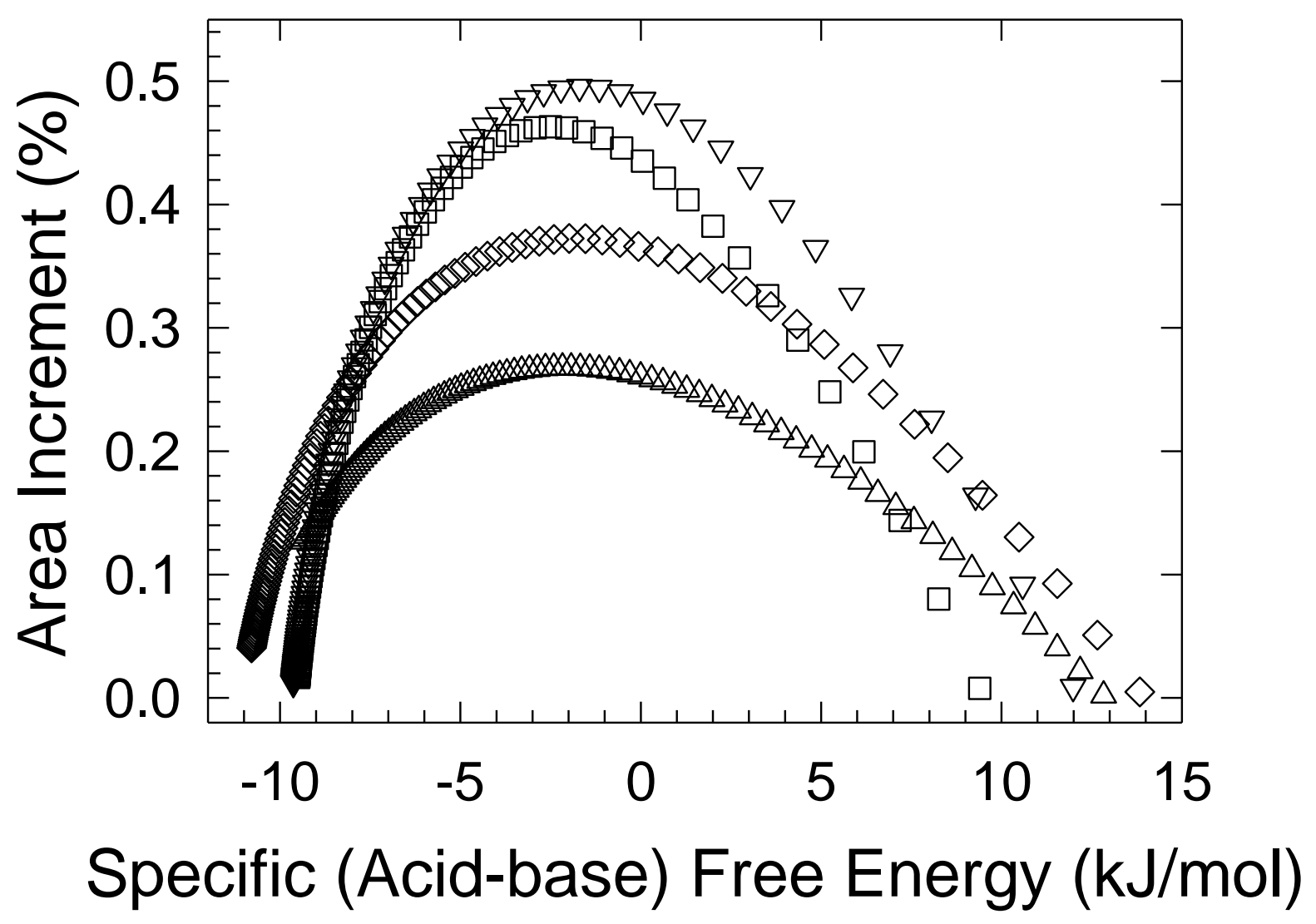

Figure 6. Specific acid-base free energy distributions of studied HA powder as observed for selected wetting probes: triangle down - ethyl acetate, square - acetone, diamond - acetonitrile, triangle up - ethanol. 


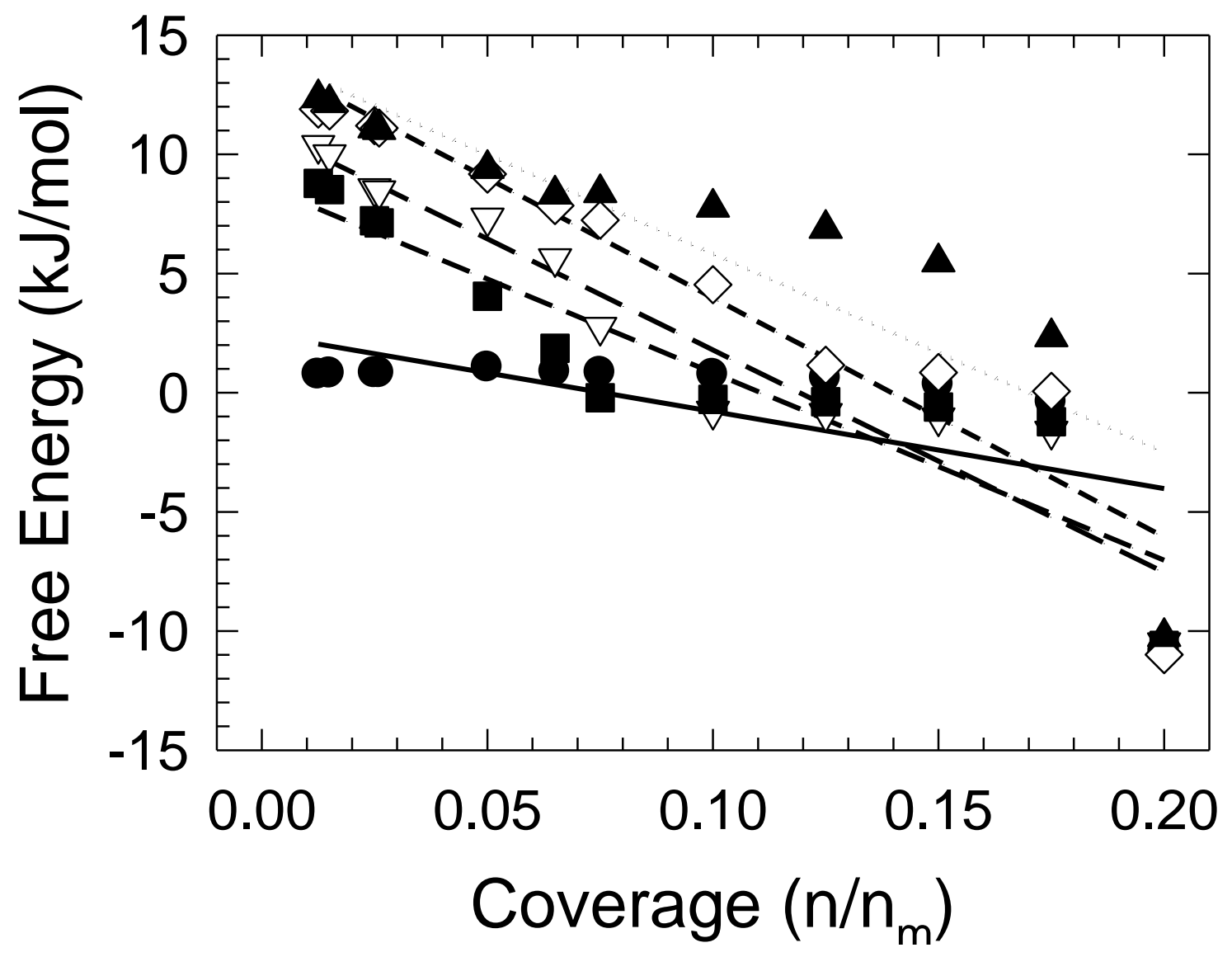

Figure 7. Coverage dependence of free energy profiles of studied HA powder as observed for selected wetting probes: full circle - dichloromethane, empty triangle down - ethyl acetate, full square - acetone, empty diamond - acetonitrile, full triangle up - ethanol. 


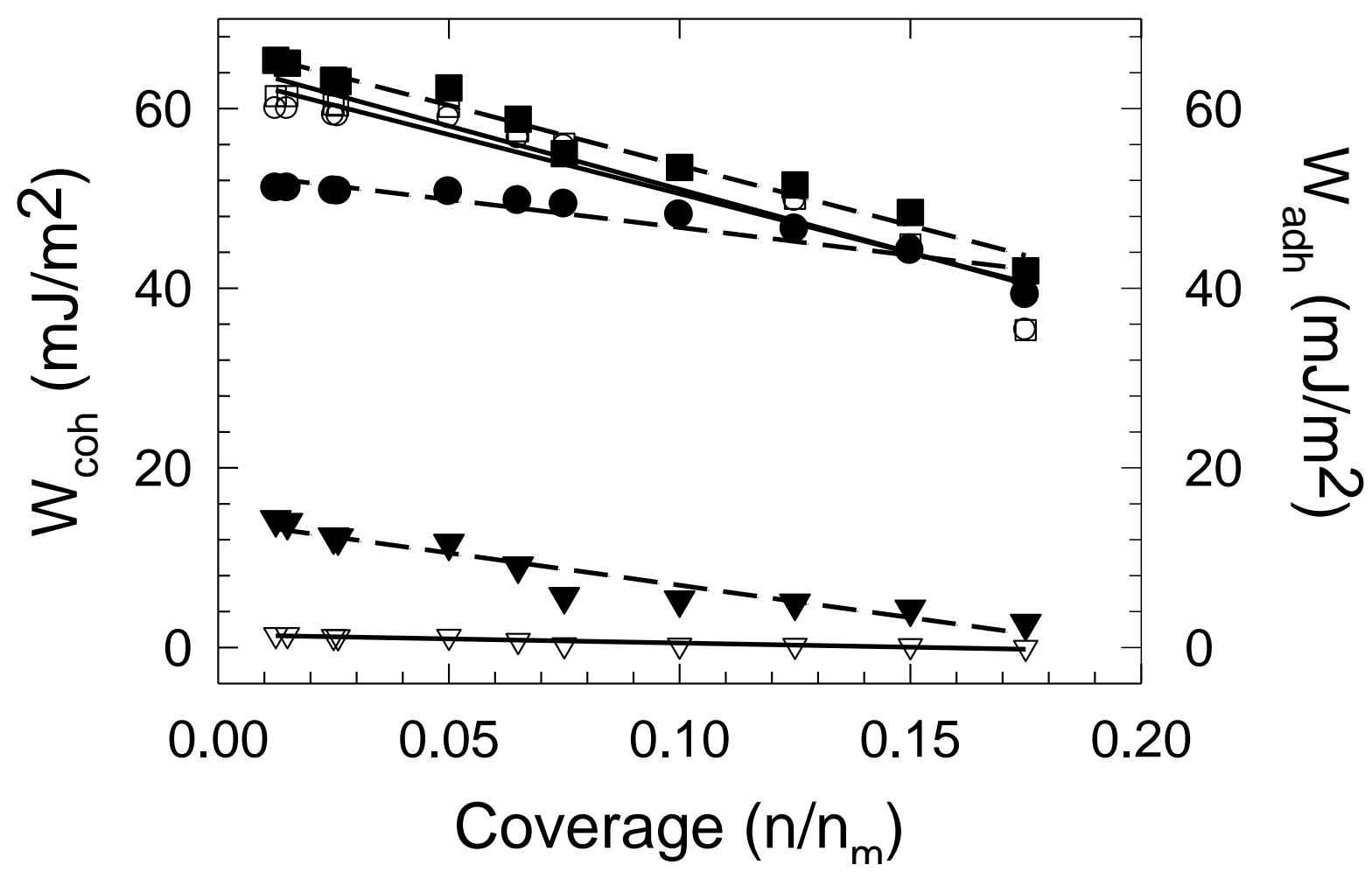

Figure 8. Work of cohesion between HA and work of adhesion and its components of water on studied HA powder at $30{ }^{\circ} \mathrm{C}$ for different coverage regimes: empty circle - work of cohesion dispersive part, empty triangle down - work of cohesion specific part, empty square - work of cohesion total, full circle - work of adhesion dispersive part, full triangle down - work of cohesion specific, full square - work of adhesion total. 


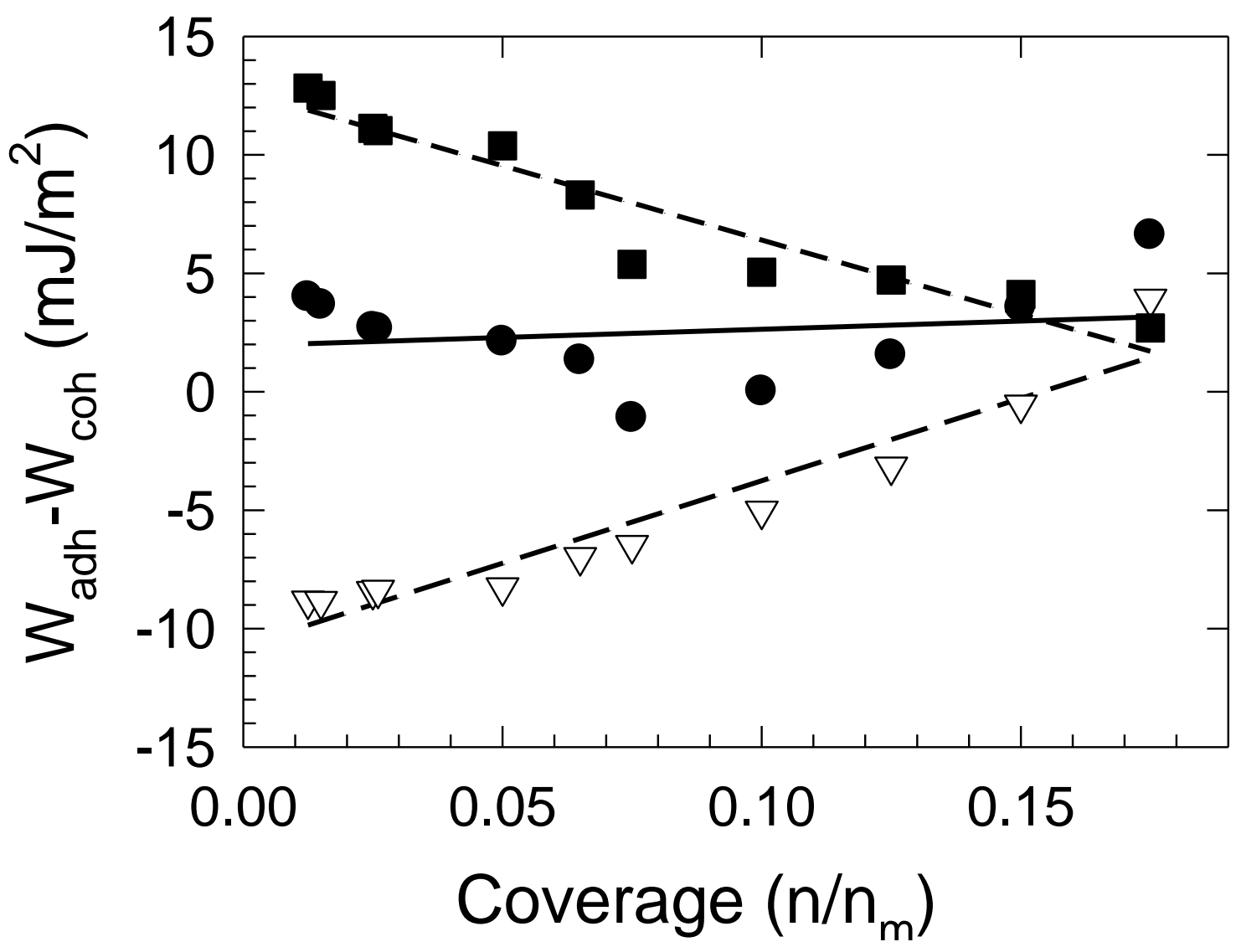

Figure 9. Difference between work of adhesion of water on HA and work of cohesion of HA and its components as a function of coverage of studied HA powder at $30^{\circ} \mathrm{C}$ : full circle - total, empty triangle down - dispersive, full square - specific. 


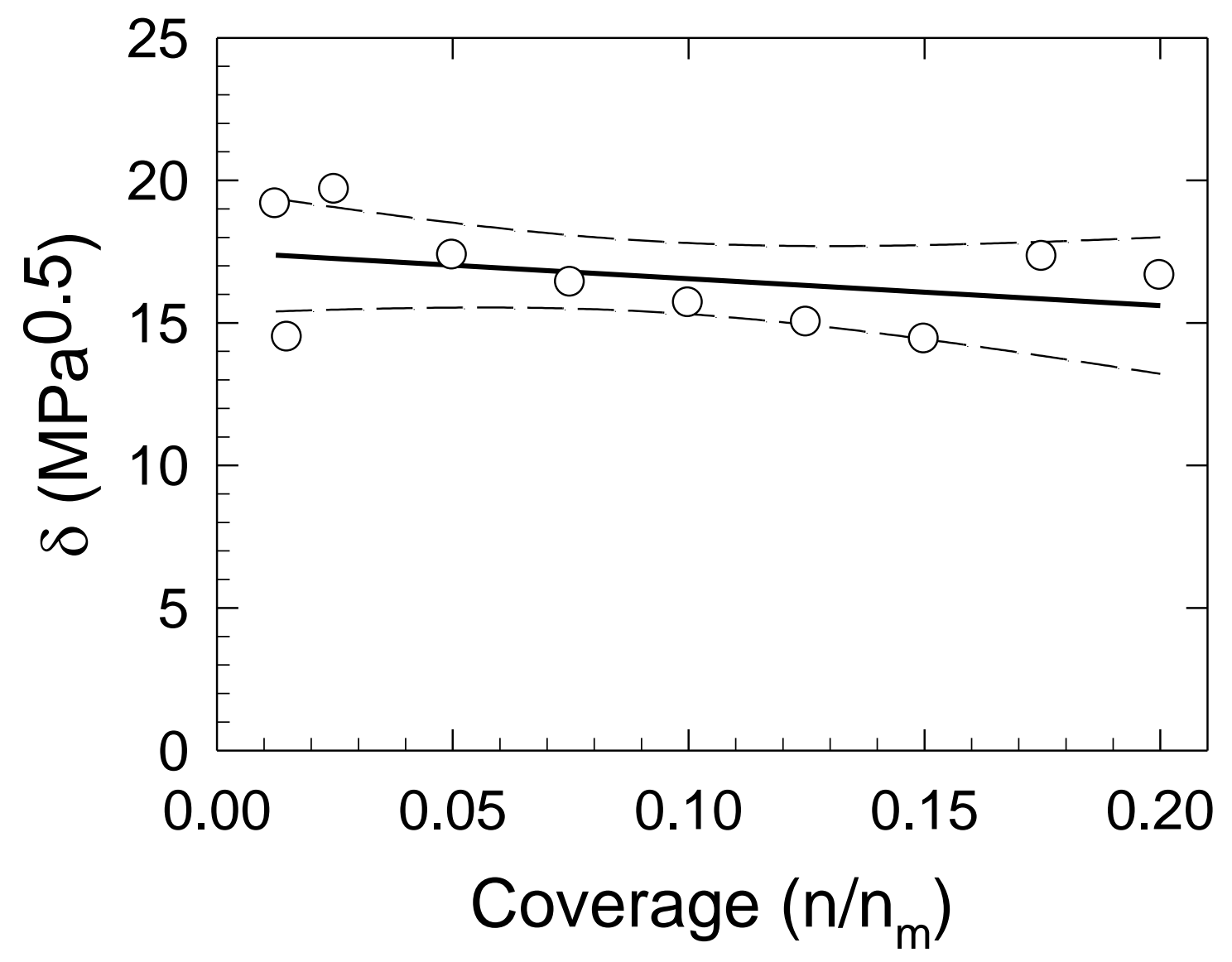

Figure 10. Surface coverage dependence of the Hildebrand's solubility parameter $\delta$ of studied HA powder. Full line - linear regression, dashed line $-95 \%$ confidence interval. 